\title{
Bernoulli potential in type-I and weak type-II superconductors: Surface charge
}

\author{
P. Lipavský ${ }^{1}$, K. Morawetz ${ }^{2,3}$, J. Koláček ${ }^{1}$, J. J. Mareš ${ }^{1}$, E. H. Brandt ${ }^{4}$, M. Schreiber ${ }^{2}$ \\ ${ }^{1}$ Institute of Physics, Academy of Sciences, Cukrovarnická 10, 16253 Prague 6, Czech Republic \\ ${ }^{2}$ Institute of Physics, Chemnitz University of Technology, 09107 Chemnitz, Germany \\ ${ }^{3}$ Max-Planck-Institute for the Physics of Complex Systems, Noethnitzer Str. 38, 01187 Dresden, Germany \\ ${ }^{4}$ Max-Planck-Institute for Metal Research, D-70506 Stuttgart, Germany
}

The electrostatic potential close to the surface of superconductors in the Meissner state is discussed. We show that beside the Bernoulli potential, the quasiparticle screening, and the thermodynamic contribution due to Rickayzen, there is a non-local contribution which is large for both type-I and weak type-II superconductors.

\section{HISTORICAL REVIEW}

The electrostatic potential in superconductors is known already for seven decades. Within the pre-London approaches based on the ideal charged liquid, the electrostatic potential was assumed to balance the Lorentz and the inertial forces acting on diamagnetic currents. ${ }^{1}$ The current $\mathbf{j}=e n \mathbf{v}$ flows along the surface and its amplitude falls off exponentially from the surface into the bulk. Similarly the screened magnetic field decays. The balancing electrostatic potential was found to be of the Bernoulli type, $e \varphi=-\frac{1}{2} m v^{2}$, therefore it falls off on the scale of half the London penetration depth. From the Poisson equation,

$$
-\epsilon_{0} \nabla^{2} \varphi=\rho,
$$

one finds that this corresponds to a charge accumulated in the layer penetrated by the magnetic field. To maintain the charge neutrality, the accumulated bulk charge has to be accompanied by the opposite surface charge. This picture has been confirmed by the London theory. ${ }^{2}$

\section{A. Advanced London-type approaches}

The electrostatic potential equals the Bernoulli potential only at zero temperature when all electrons are in the superconducting condensate. At finite temperatures, a part $n_{n}$ of electrons remains in the normal state, while the rest $n_{s}=n-n_{n}$ contributes to the supercurrent, $\mathbf{j}=e n_{s} \mathbf{v}$. The electric force acting on normal electrons is transferred to the condensate via a mechanism which reminds one of the fountain effect in superfluid helium. As a consequence, the electrostatic potential reduces to $e \varphi=-\frac{n_{s}}{n} \frac{1}{2} m v^{2}$. This reduction of the potential derived by van Vijfeijken and Staas $^{3}$ has a somehow confusing name: the quasiparticle screening. It should be noted that the electrostatic potential caused by currents in superconductors is traditionally called the Bernoulli potential in spite of the quasiparticle screening and other nonBernoulli contributions found later.

Jakeman and Pike recovered the result of van Vijfeijken and Staas from the static and the classical limit of the time-dependent Ginzburg-Landau (GL) theory. ${ }^{4}$ Unlike previous studies, their approach includes the real screening due to spatial distribution of the charge, therefore it describes the surface charge as a space charge localized on the scale of the Thomas-Fermi screening length. This picture of the surface charge was accepted for a long time as it looks natural and seems to be justified from the semi-microscopic theory.

Later studies of the electrostatic potential did not readdress the question of the surface charge but attempted to overcome the hydrodynamic picture common to all the above mentioned studies. Naturally, they focused on a detailed description of the Bernoulli potential on the scale of the London penetration depth.

Adkins and Waldram recovered the Bernoulli potential from the BCS theory for the system at zero temperature. ${ }^{5}$ They also indicated that at finite temperatures one should expect a contribution which depends on the band structure.

The predicted contribution has been evaluated by Rickayzen. ${ }^{6}$ He used the thermodynamic approach which yields

$$
e \varphi=-\frac{\partial n_{s}}{\partial n} \frac{1}{2} m v^{2}
$$

The density dependence of the condensate density can be evaluated either from the BCS theory leading to the result of Adkins and Waldram or from the two-fluid relation $n_{s} \approx n\left(1-\frac{T^{4}}{T_{c}^{4}}\right)$. The latter gives a simple formula

$$
e \varphi=-\frac{n_{s}}{n} \frac{1}{2} m v^{2}-4 \frac{n_{n}}{n} \frac{\partial \ln T_{c}}{\partial \ln n} \frac{1}{2} m v^{2} .
$$

The first term balances the Lorentz and inertial forces, while the second reflects the pairing. We will call terms proportional to $\frac{\partial \ln T_{c}}{\partial \ln n}$ the thermodynamic correction.

In the next decade, the interest in the electrostatic potential in superconductors shifted towards nonequilibrium situations, see e.g. Ref. 7. It should be noted that the Bernoulli potential introduced within the theory of non-equilibrium systems is not the same as the one we discuss. Within the non-equilibrium theory the term Bernoulli potential is used for the velocity-dependent part of the energy of Cooper pairs. Only in equilibrium, 
the velocity-dependent energy agrees with the electrostatic potential we discuss here.

\section{B. GL approaches - local}

New studies of the Bernoulli potential in equilibrium have been stimulated by the question of the vortex charge. Khomskii et al. ${ }^{8,9}$ have used the idea of van der Marel ${ }^{10}$ to derive a simple estimate of the electrostatic potential in terms of the superconducting gap. The obtained formula corresponds to the thermodynamic correction of Rickayzen with the original balance term being omitted. While the validity of the approach is restricted to temperatures close to $T_{c}$, it has the advantage, however, that it can be applied within the GL theory. Blatter et al. ${ }^{11}$ have employed this estimate to predict a possible electrostatic field above the surface of the superconductor with the vortex lattice perpendicular to it.

An opposite limit represents the treatment of the vortex charge by LeBlanc. ${ }^{12}$ He considers only the balance term omitting the thermodynamic correction completely. As one can see from Rickayzen's formula, this approximation is limited to the region of low temperatures.

\section{GL approaches - non-local}

All the above mentioned theories have in common that the electrostatic potential is a local function of the classical kinetic energy or of the gap. In other words, they neglect the gradient of the condensate. The non-local corrections within the GL theory have been proposed in Ref. 13. It turned out that the non-local corrections are taken into account if the classical kinetic energy in the balance equation is replaced by its quantum-mechanical counterpart.

For our next discussion it is important that the nonlocal contributions can be rearranged into a local form. ${ }^{13}$ Indeed, within the GL theory the sum of the kinetic energy and the GL potential is zero which allows us to express the balance term via the local GL potential. Note that this rearrangement is possible only within the nonlocal theory, because gradients of the condensate density $n_{s}=2|\psi|^{2}$, where $\psi$ is the GL wave function, provide important contributions to the quantum kinetic energy, in particular close to the surface.

In Ref. 13, the quasiparticle screening and the thermodynamic correction have been neglected, the complete theory has been presented in Ref. 14. The detailed derivation together with estimates of material parameters and the numerical treatment of the Abrikosov vortex lattice in niobium at various temperatures can be found in Ref. 15. Again, all contributions including the kinetic energy can be expressed in a local relation $\varphi\left[|\psi|^{2}\right]$.

The non-local form of the Bernoulli potential requires to reconsider the picture of surface charges. It is para- doxical that the possibility to rearrange the non-local corrections into a local function plays the crucial role. As noticed already by Yampolskii et al., ${ }^{16}$ if the electrostatic potential is of the form $\varphi\left[|\psi|^{2}\right]$, the GL boundary condition, $\nabla \psi=0$ in the direction normal to the surface, implies that no surface charge is needed. Indeed, at the surface $\nabla \varphi=\frac{\partial \varphi}{\partial \psi} \nabla \psi=0$. The electric field thus vanishes at the surface which is a sufficient condition for the charge neutrality.

\section{Plan of the paper}

Apparently, there is no surface charge on the scale of the Thomas-Fermi screening length. The electric field due to the dipole between the surface charge and the bulk charge was supposed to balance the Lorentz force, therefore one has to ask the question how the balance of forces looks if the surface charge is absent.

The charge profile in the vicinity of the surface has been numerically studied in Ref. 17 . This study was limited to thin slabs and type-II superconductors, its results indicate, however, that the charge profile at the surface tends to organize into two layers - a narrow surface charge and a thicker bulk charge.

In this paper we discuss the charge and potential profile at the surface of a semi-infinite superconductor in the Meissner state. In the next section we shortly describe the surface charge within the GL theory. In section III we discuss the limit of a weak magnetic field, where all results can be derived analytically. We will show that the Bernoulli potential at the surface differs from the value predicted by Rickayzen. The correction has the form of a multiplicative factor that approaches unity for the extreme type-II superconductor. Numerical results on strong magnetic fields are presented in section IV revealing a nonlinear mechanism which, however, is found to be very small. The conclusions and outlook are presented in section $\mathrm{V}$.

\section{SURFACE CHARGE WITHIN THE GINZBURG-LANDAU TYPE THEORY}

A typical profile with the charge distribution close to the planar surface and homogeneous external magnetic fields is shown in Fig. 1. The profile has been obtained by a numerical solution of the GL equation

$$
-\frac{\hbar^{2} \nabla^{2}}{2 m^{*}} \psi+\frac{e^{* 2} \mathbf{A}^{2}}{2 m^{*}} \psi-\frac{\gamma T_{c}^{2}}{2 n}\left(1-\frac{t^{2}}{\sqrt{1-\frac{2}{n} \psi^{2}}}\right) \psi=0,
$$

where $n$ is the density of pairable electrons, $\gamma$ is the linear coefficient of the specific heat, $m^{*}=2 m$ and $e^{*}=2 e$ are the mass and charge of the Cooper pair, and $t=T / T_{c}$ is 
the temperature on the scale of the critical temperature. In the assumed geometry one can use the London gauge in which the GL wave function is real.

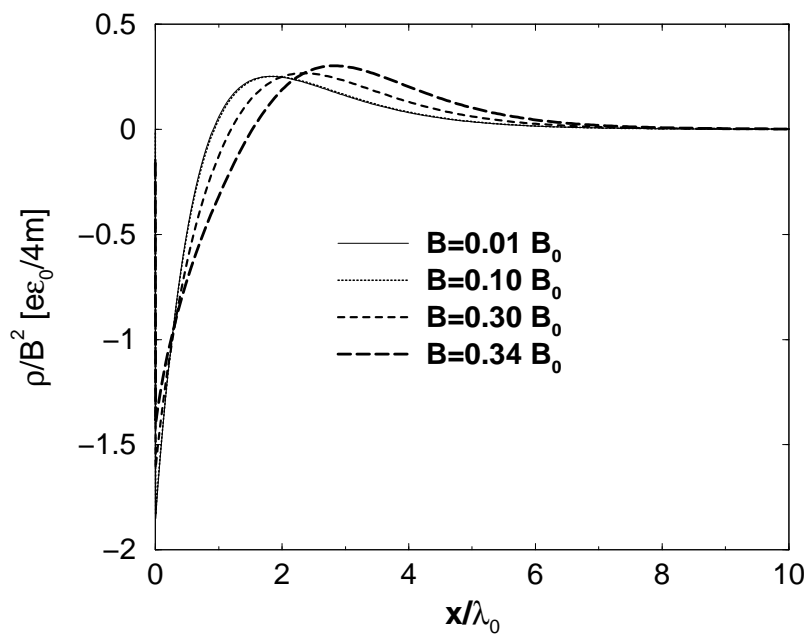

FIG. 1. The charge profile close to the surface for 4 different magnetic fields. The magnetic field is given in dimensionless units proportional to the magnetic flux quanta, $B_{0}=\Phi_{0} /\left(2 \pi \lambda_{0}^{2}\right)=e \mu_{0} n \hbar / 2 m$. The coordinate $x$ is measured in units of the London penetration depth $\lambda_{0}$ at zero temperature. The assumed temperature $t=T / T_{c}=0.9$ yields the penetration depth $\lambda=1.7 \lambda_{0}$. The charge is scaled with $B^{2}$ so that the two lowest magnetic fields $B=0.01 B_{0}$ and $B=0.1 B_{0}$ give nearly identical curves. The magnetic field $B=0.34 B_{0}$ is close to the critical value. The GL parameter $\kappa_{\mathrm{GL}}=1$ corresponds to weak type-II superconductors.

Equation (4) has been proposed by Bardeen as an extension to low temperatures. ${ }^{18}$ In fact, Bardeen arrived to this equation independently adding the quantum kinetic energy to the Gorter-Casimir two-fluid model. Close to the critical temperature the GL function has a small magnitude $2 \psi^{2} \ll n$ which allows one to expand the square$\operatorname{root} 1 / \sqrt{1-\frac{2}{n} \psi^{2}} \approx 1+\frac{1}{n} \psi^{2}$, and one finds the customary GL equation with parameters $\alpha=\gamma T_{c}^{2}\left(t^{2}-1\right) / 2 n$ and $\beta=\gamma T_{c}^{2} t^{2} / n^{2}$.

The vector potential $\mathbf{A}$ is given by the Maxwell equation

$$
\nabla \times \nabla \times \mathbf{A}=\mu_{0} \frac{e^{* 2}}{m^{*}} \psi^{2} \mathbf{A} .
$$

Equations (4) and (5) are solved together with $\gamma$ and $T_{c}$ kept constant.

From the GL wave function $\psi$ we evaluate the Bernoulli potential $^{15}$

$$
\begin{aligned}
e \varphi-\lambda_{\mathrm{TF}}^{2} \nabla^{2} e \varphi= & -\frac{\gamma T_{c}^{2}}{2 n^{2}}\left(1-\frac{t^{2}}{\sqrt{1-\frac{2}{n} \psi^{2}}}\right) \psi^{2} \\
& +\frac{\psi^{2}}{2 n} \frac{\partial \gamma T_{c}^{2}}{\partial n}+\frac{t^{2} T_{c}^{2}}{2} \frac{\partial \gamma}{\partial n} \sqrt{1-\frac{2}{n} \psi^{2}}
\end{aligned}
$$

The density dependence of $\gamma$ and $T_{c}$ has been estimated from the experimental specific heat, theoretical freeelectron density of states, and the McMillan formula ${ }^{19}$, see Appendix in Ref. 15. The Thomas-Fermi screening length, $\lambda_{\mathrm{TF}}^{2}=\frac{\pi^{2} k_{B}^{2} \epsilon_{0}}{\gamma e^{2}}$, is very small and its contribution can be neglected, because the general integral which is proportional to $\exp \left(-x / \lambda_{\mathrm{TF}}\right)$ is zero since no surface charge forms on this scale.

In the final step the charge profile is evaluated from the Poisson equation (1).

\section{LOW MAGNETIC FIELDS}

In weak magnetic fields the GL wave function is only little perturbed from its bulk value $\psi=\psi_{\infty}+\delta \psi$ with $\delta \psi \ll \psi_{\infty}$. In this limit the charge profile can be solved analytically.

In the Maxwell equation (5) the vector potential is a small quantity so that the perturbation of the wave does not contribute in lowest order. The vector potential can thus be solved taking $\psi \approx \psi_{\infty}=\sqrt{n\left(1-t^{4}\right) / 2}$. For both types of superconductors, this results in a simple exponential decay of the vector potential,

$$
A_{x} \approx B \lambda \mathrm{e}^{-x / \lambda}
$$

on the scale of the London penetration depth $\lambda=$ $\lambda_{0} / \sqrt{1-t^{4}}$, with $\lambda_{0}^{2}=2 m^{*} /\left(\mu_{0} e^{* 2} n\right)$. Here $B$ is the value of the magnetic field at the surface at $x=0$.

\section{A. GL wave function}

The linear approximation of the GL equation (4) reads

$$
\frac{\hbar^{2} \nabla^{2}}{2 m^{*}} \delta \psi-\frac{\gamma T_{c}^{2} t^{2}}{n^{2}}\left(1-\frac{2}{n} \psi_{\infty}^{2}\right)^{-\frac{3}{2}} \psi_{\infty}^{2} \delta \psi=\frac{e^{* 2} \mathbf{A}^{2}}{2 m^{*}} \psi_{\infty}
$$

which can be expressed in terms of the GL coherence length $\xi^{2}=2 \hbar^{2} n t^{4} /\left(m^{*} \gamma T_{c}^{2}\left(1-t^{4}\right)\right)$ as

$$
\nabla^{2} \delta \psi-\frac{2}{\xi^{2}} \delta \psi=\frac{e^{* 2}}{\hbar^{2}} \psi_{\infty} \lambda^{2} B^{2} \mathrm{e}^{-2 x / \lambda}
$$

The solution of (9) is composed from the particular integral with the decay on the scale of the London penetration depth and the general integral decaying on the scale of the GL coherence length,

$$
\delta \psi=\psi_{\lambda} \mathrm{e}^{-2 x / \lambda}+\psi_{\xi} \mathrm{e}^{-\sqrt{2} x / \xi}
$$

The general integral of (9) can also include a growing term $\propto \exp (\sqrt{2} x / \xi)$. This term is excluded, because the perturbation asymptotically vanishes in the bulk, $\delta \psi \rightarrow 0$ for $x \rightarrow \infty$. 
From the GL boundary condition, $\nabla \delta \psi=0$ at $x=0$, we find that the two amplitudes are linked by

$$
\psi_{\xi}=-\frac{\sqrt{2}}{\kappa} \psi_{\lambda},
$$

where $\kappa=\lambda / \xi$. We note that within Bardeen's extension of the GL theory, the ratio $\lambda / \xi$ depends on the temperature, $\kappa=\kappa_{\mathrm{GL}} / t^{2}$, where $\kappa_{\mathrm{GL}}=\frac{m^{*} T_{c}}{n e^{*} \hbar} \sqrt{\frac{\gamma}{\mu_{0}}}$ is the ordinary GL parameter defined at $T_{c}$. The diverging effective GL parameter at low temperatures shows that within Bardeen's approximation any system behaves as a type-II superconductor at sufficiently low temperatures. This feature contrasts with the linearly decreasing effective GL parameter one finds close to the upper critical magnetic field. ${ }^{15}$. Of course, the strong temperature dependence of $\kappa$ is rather an artifact of the model. Since $\kappa$ is the most important quantity of the GL theory, one can simply take $\lambda$ and $\kappa$ as input parameters.

The amplitude of the particular integral is obtained from the GL equation (9) as

$$
\psi_{\lambda}=\psi_{\infty} \frac{\frac{e^{* 2}}{\hbar^{2}} \lambda^{2} B^{2}}{\frac{4}{\lambda^{2}}-\frac{2}{\xi^{2}}}=\psi_{\infty} \frac{e^{* 2} \lambda^{4} B^{2}}{2 \hbar^{2}} \frac{1}{2-\kappa^{2}} .
$$

The GL wave function from (10-12) results in

$$
\delta \psi=\frac{\psi_{\infty}}{2-\kappa^{2}} \frac{e^{* 2} \lambda^{4} B^{2}}{2 \hbar^{2}}\left(\mathrm{e}^{-2 x / \lambda}-\frac{\sqrt{2}}{\kappa} \mathrm{e}^{-\sqrt{2} x / \xi}\right) .
$$

\section{B. Bernoulli potential}

Finally, we take the linear approximation of the Bernoulli potential (6) in $\delta \psi$. Since the asymptotic value of the potential $\varphi_{\infty}=\varphi\left[\left|\psi_{\infty}\right|^{2}\right]$ is not essential, we focus on the perturbation caused by the magnetic field

$$
e \delta \varphi-\lambda_{\mathrm{TF}}^{2} \nabla^{2} e \delta \varphi=C \delta \psi .
$$

In terms of $\lambda$ and $\kappa$ the coefficient $C$ reads

$$
C=\frac{\hbar^{2} \kappa^{2}}{2 m^{*} \lambda^{2} \psi_{\infty}}\left(1-t^{4}+4 t^{4} \frac{\partial \ln T_{c}}{\partial \ln n}\right) .
$$

A general integral which decays into the bulk is of the form

$$
e \delta \varphi=e \delta \varphi_{\mathrm{TF}} \mathrm{e}^{-x / \lambda_{\mathrm{TF}}}+C_{\lambda} \psi_{\lambda} \mathrm{e}^{-2 x / \lambda}+C_{\xi} \psi_{\xi} \mathrm{e}^{-\sqrt{2} x / \xi} .
$$

From (14) one finds the amplitudes

$$
\begin{aligned}
C_{\lambda} & =\frac{C}{1-4 \frac{\lambda_{\mathrm{TF}}^{2}}{\lambda^{2}}}, \\
C_{\xi} & =\frac{C}{1-2 \frac{\lambda_{\mathrm{TF}}^{2}}{\xi^{2}}} .
\end{aligned}
$$

The amplitude of the general integral is given by the charge neutrality, which requires $\nabla \delta \varphi=0$ at $x=0$. From (16) follows

$$
e \delta \varphi_{\mathrm{TF}}=-2 \frac{\lambda_{\mathrm{TF}}}{\lambda} C_{\lambda} \psi_{\lambda}-\sqrt{2} \frac{\lambda_{\mathrm{TF}}}{\xi} C_{\xi} \psi_{\xi} .
$$

The relative amplitudes proportional to $\lambda_{\mathrm{TF}} / \lambda$ and $\lambda_{\mathrm{TF}} / \xi$ are not sufficiently small to be neglected. The charge density is given by the second derivatives so that one obtains relative amplitudes of the charge density at the surface proportional to $\lambda / \lambda_{\mathrm{TF}}$ or to $\xi / \lambda_{\mathrm{TF}}$. The surface charge is an integral, i.e. the contribution in question is proportional to the amplitude at the surface multiplied with the Thomas-Fermi length. According to condition (19) all charges can have comparable values.

To prove that the screening on the scale of the ThomasFermi length $\lambda_{\mathrm{TF}}$ and the related surface charge can be neglected, one has to employ the GL boundary condition $\nabla \delta \psi=0$ at $x=0$. Using (11) one obtains

$$
e \delta \varphi_{\mathrm{TF}}=-\frac{\lambda_{\mathrm{TF}}^{3}}{\lambda^{3}} \frac{4\left(2-\kappa^{2}\right)}{1-2 \kappa^{2} \lambda_{\mathrm{TF}}^{2} / \lambda^{2}} C_{\lambda} \psi_{\lambda}
$$

so that the general integral is proportional to the cube of the small ratio $\lambda_{\mathrm{TF}} / \lambda$. Accordingly, the charge due to the general integral is smaller by a factor $\lambda_{\mathrm{TF}}^{2} / \lambda^{2}$ than the charge created by the particular integral. In the following we neglect this contribution for simplicity of notation. Within the same level of accuracy we take $C_{\lambda}=C$ and $C_{\xi}=C$.

Now we are ready to evaluate the electrostatic potential. Using (11) and (12) we obtain from (16)

$$
\begin{aligned}
e \delta \varphi= & -\frac{B^{2}}{2 \mu_{0} n}\left(1+\frac{4 t^{4}}{1-t^{4}} \frac{\partial \ln T_{c}}{\partial \ln n}\right) \\
& \times \frac{1}{1-\frac{2}{\kappa^{2}}}\left(\mathrm{e}^{-2 x / \lambda}-\frac{\sqrt{2}}{\kappa} \mathrm{e}^{-\sqrt{2} x / \xi}\right) .
\end{aligned}
$$

This linearized relation extends Rickayzen's formula to type-I and weak type-II superconductors.

Let us first take a look at the extreme type-II superconductor, $\kappa \gg 1$, for which Rickayzen's formula is recovered. For the assumed real GL wave function, the current is proportional to the vector potential $\mathbf{j}=$ $\frac{e^{* 2}}{m^{*}} \psi_{\infty}^{2} \mathbf{A}=\frac{e^{2}}{m} n_{s} \mathbf{A}$. With the velocity defined via the current, $\mathbf{j}=e^{*} \psi_{\infty}^{2} \mathbf{v}=e n_{s} \mathbf{v}$, one finds from (7) the relation between the magnetic pressure and the kinetic energy $\mathrm{e}^{-2 x / \lambda} \frac{B^{2}}{2 \mu_{0} n}=\frac{n_{s}}{n} \frac{1}{2} m v^{2}$. Equation (21) yields then the Bernoulli potential as

$$
\begin{aligned}
e \delta \varphi= & -\frac{1}{2} m v^{2}\left(\frac{n_{s}}{n}+4 \frac{n_{n}}{n} \frac{\partial \ln T_{c}}{\partial \ln n}\right) \\
& \times \frac{1}{1+\frac{\sqrt{2}}{\kappa}}\left(1+\frac{\mathrm{e}^{(1-\kappa / \sqrt{2}) 2 x / \lambda}-1}{\left.1-\frac{\kappa}{\sqrt{2}}\right) .} .\right.
\end{aligned}
$$


We have used $n_{s}=n\left(1-t^{4}\right)$ and $n_{n}=n t^{4}$ to express the temperature dependence in terms of the condensate fraction.

One can see that Rickayzen's formula (3) holds for extreme type-II superconductors, $\kappa \rightarrow \infty$. In this limit the general integral has a vanishingly small amplitude and the factor $1 /\left(1+\frac{\sqrt{2}}{\kappa}\right)$ goes to unity. For finite $\kappa$ the general integral deforms the profile of the potential and the particular integral has a reduced amplitude.

Within Bardeen's extension of the GL theory, Rickayzen's formula is always recovered at low temperatures as $\kappa$ diverges with $1 / t^{2}$. Since Rickayzen's formula approaches the plain Bernoulli potential for $t \rightarrow 0$, the presented derivation also reproduces London's result.

\section{Behavior for finite $\kappa$}

As already shown, for the extreme type-II superconductor, $\kappa \rightarrow \infty$, the general integral vanishes as $1 / \kappa$ and far from the surface it decays faster than the particular integral. The Bernoulli potential thus extends on the scale $\lambda / 2$.

For the finite GL parameter in the region $\kappa>\sqrt{2}$ the overall picture is rather similar. The general integral is appreciable only close to the surface so that the scale on which the Bernoulli potential extends is still $\lambda / 2$. Besides, the amplitude of the particular integral is enhanced by the factor $1 /\left(1-2 / \kappa^{2}\right)$.

For the region $\kappa<\sqrt{2}$, the role of the general and particular integrals are reversed. First of all, the amplitude of the general integral is larger than the amplitude of the particular integral. Since the general integral also decays more slowly in this case, the Bernoulli potential is dominated by the general integral and extends on the scale $\xi / \sqrt{2}$. The reversed role is also supported by the fact that the signs of both parts are opposite as compared to the case $\kappa>\sqrt{2}$. This sign reversal appears due to the enhancement factor $1 /\left(1-2 / \kappa^{2}\right)$.

Apparently, both components of $\delta \varphi$ diverge as $\kappa$ approaches $\sqrt{2}$. Their sum remains regular, however. For this particular case the scale of both contributions is identical, namely $\lambda / 2$. It is advantageous to use expression (21) to obtain the asymptotic form of the Bernoulli potential

$$
\lim _{\kappa \rightarrow \sqrt{2}} e \delta \varphi=-\frac{m}{4} v^{2}\left(\frac{n_{s}}{n}+4 \frac{n_{n}}{n} \frac{\partial \ln T_{c}}{\partial \ln n}\right)\left(1+\frac{2 x}{\lambda}\right) .
$$

The Bernoulli potentials for different values of the magnetic field are shown in Fig. 2. The limit of low magnetic fields agrees with the analytical formula (22), of course. One can see that for the two lowest fields $B=0.01$ and $B=0.1$, the potential keeps its profile while its magnitude scales with $B^{2}$. For magnetic fields close to the critical value $B_{c 2} \approx 0.34$, the linear theory does not apply.

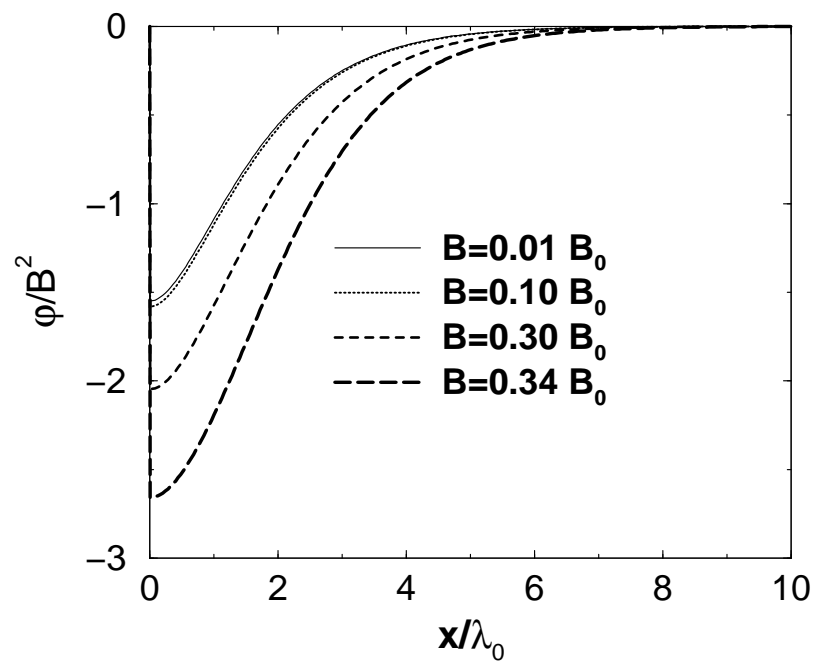

FIG. 2. Bernoulli potential as a function of the distance from the surface for 4 different magnetic fields. The parameters are the same as in Fig. 1.

\section{Charge profile}

The charge density results from the Bernoulli potential (21) and the Poisson equation (1) as

$$
\rho=\frac{2 e \epsilon_{0} B^{2}}{m\left(1-\frac{2}{\kappa^{2}}\right)}\left(\frac{n_{s}}{n}+4 \frac{n_{n}}{n} \frac{\partial \ln T_{c}}{\partial \ln n}\right)\left(\mathrm{e}^{-2 x / \lambda}-\frac{\kappa}{\sqrt{2}} \mathrm{e}^{-\sqrt{2} x / \xi}\right) .
$$

Note that the relative amplitude of the two contributions is reciprocal to the relative amplitude of the potentials. Integrating from zero to infinity one can check that the total charge is zero, as it is required by the charge neutrality.

The resulting charge density is small compared to the total density of electrons $e n$. The ratio of the amplitude of (24) to the total electron density can be written as

$$
\frac{\rho}{e n} \approx \frac{\epsilon_{0} B^{2}}{m n}=\frac{B^{2}}{\mu_{0} n} \frac{1}{m c^{2}}
$$

Even for upper estimates of the magnetic field by the critical value, $B \approx B_{c}$, the factor (25) is extremely small. For niobium $B^{2} / \mu_{0}=\frac{1}{2} \gamma T_{c}^{2}=3.2 \times 10^{4} \mathrm{~J} / \mathrm{m}^{3}$, while $m c^{2} \approx 10^{-13} \mathrm{~J}$. This suggests that the maximum charge density will be of the order of $10^{18}$ electrons per cubic meter. This is by ten orders of magnitude smaller than the electron density of niobium $n=2.2 \times 10^{28} \mathrm{~m}^{-3}$. This small value of the accumulated or depleted charge justifies to ignore the charge profile in the Maxwell equation (5) and the change of material parameters like $\gamma, n$ and $T_{c}$ in the GL equation (4). 
Similarly to the potential, the charge profile reflects two scales, $\lambda / 2$ and $\xi / \sqrt{2}$. Before we analyze its properties for various values of the GL parameter $\kappa$, we want to discuss a few general features. First, the surface value of the charge, obtained for $x=0$ in (24), is always negative, i.e. the charge carriers are depleted. We call the layer of the negative charge the surface charge. Second, in the bulk sufficiently far from the surface the charge is positive, i.e., the charge carriers are accumulated. We call the region of positive charge the bulk charge. Third, the width $w$ of the surface is given by $\rho(w)=0$. From (24) follows

$$
w=\frac{\lambda}{2} \frac{\ln \frac{\kappa}{\sqrt{2}}}{\frac{\kappa}{\sqrt{2}}-1} .
$$

In the extreme type-II superconductor, $\kappa \rightarrow \infty$, the surface charge is formed by the contribution on the scale of the GL coherence length $\xi$. The width of the surface goes to a value $w \rightarrow \frac{\xi}{\sqrt{2}} \ln \frac{\kappa}{\sqrt{2}}$. The bulk charge extends on the scale of the London penetration depth as it is known from the classical picture.

For the limiting case $\kappa \rightarrow \sqrt{2}$ the width of the surface charge is $\lambda / 2$. The charge density has the profile

$$
\rho=-\frac{e \epsilon_{0} B^{2}}{m}\left(\frac{n_{s}}{n}+4 \frac{n_{n}}{n} \frac{\partial \ln T_{c}}{\partial \ln n}\right) \mathrm{e}^{-2 x / \lambda}\left(1-\frac{2 x}{\lambda}\right) .
$$

Note the different sign inside the last parentheses compared to the Bernoulli potential (23). Since $\lambda / 2=\xi / \sqrt{2}$ one cannot associate the surface and the bulk charge to the GL coherence length $\xi$ or to $\lambda$.

For $\kappa<\sqrt{2}$, the surface charge is formed by the contribution on the scale of the London penetration depth. For $\kappa \ll \sqrt{2}$, the width of the surface charge is $w \rightarrow \frac{\lambda}{2} \ln \frac{\sqrt{2}}{\kappa}$. The bulk charge extends on the scale of the GL coherence length $\xi$ as one can see from (24).

\section{STRONG MAGNETIC FIELD}

In strong magnetic fields the GL wave function is suppressed in the vicinity of the surface which has to be accounted for in the Maxwell equation. In this case one has to face the fact that the system of equations (4) and (5) is nonlinear. We present only a few numerical solutions to point out some features typical for strong magnetic fields.

The nonlinear effects in strong magnetic fields follow from a deviation of the wave function from its bulk value. In the dimensionless representation used in Fig. 3 the bulk value is $\psi_{\infty}=\sqrt{n / 2} \sqrt{1-t^{4}} \approx 0.6$. For the lowest field $B=0.01$, the deviation is nearly zero on the scale of the graph. Also for $B=0.1$ the approximation $|\delta \psi| \ll$ $\psi_{\infty}$ is justified. For higher fields the deviation becomes appreciable.

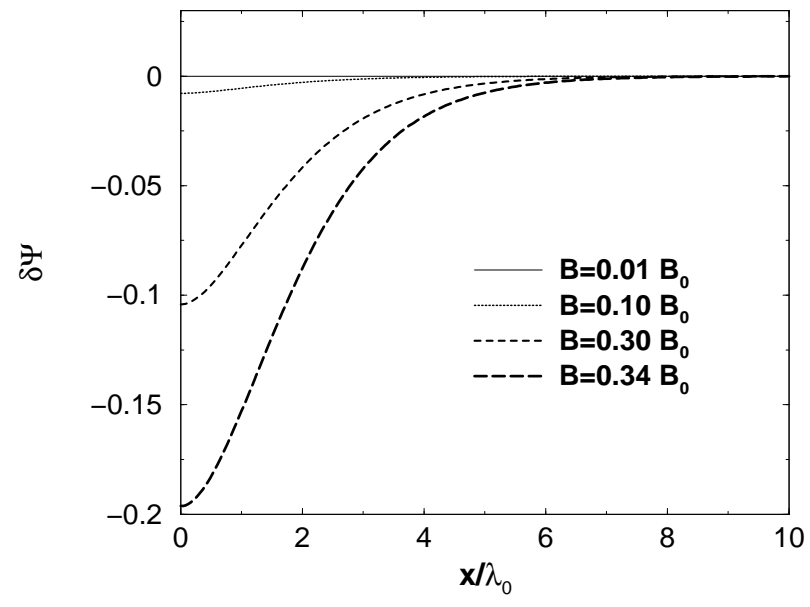

FIG. 3. The deviation of the wave function from its bulk value $\psi_{\infty}=\sqrt{n\left(1-t^{4}\right) / 2} \approx 0.6$ as a function of the distance from the surface. The parameters are the same as in Fig. 1.

The suppression of the wave function at the surface shown in Fig. 3 affects the Bernoulli potential by two different mechanisms. First, the screening current is reduced at the surface so that the magnetic field penetrates deeper into the superconductor. This feature can be seen in Fig. 2, however, it is better visible in the profile of the charge density shown in Fig. 1, as a widening of the surface layer.

Second, the thermodynamic contributions to the Bernoulli potential are nonlinear in the wave function, see (6). In contrast, the linearized approximation of the Bernoulli potential (21) or (22) does not depend on the density derivative of the linear coefficient of the specific heat, $\partial \gamma / \partial n$, included in the derivative of the condensation energy $\frac{1}{4} \gamma T_{c}^{2}$. Beyond the linearized approximation, this derivative contributes as demonstrated in Fig. 4 for parameters of niobium.

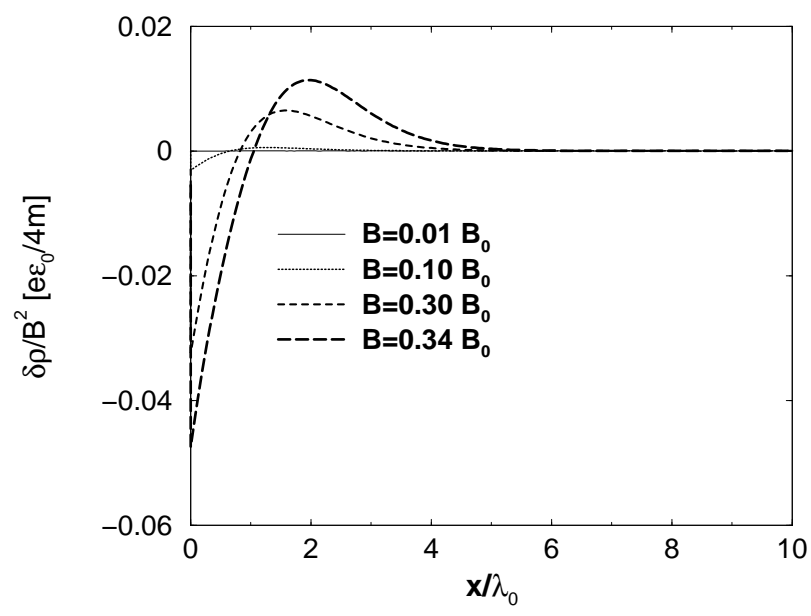

FIG. 4. The charge density which is proportional to the density dependence of the linear coefficient of the specific heat versus distance from the surface. We use the value for niobium, $\partial \ln \gamma / \partial \ln n=0.42$, which is close to the parabolic band approximation $\gamma_{\text {par }} \propto n^{1 / 3}$, i.e., $\partial \ln \gamma_{\text {par }} / \partial \ln n=1 / 3$. 
Comparing the contribution of $\partial \gamma / \partial n$ with the total charge density shown in Fig. 1, one can see that this particular nonlinear mechanism is rather small giving less than $3 \%$ of the total charge density. This small value justifies the approximations ${ }^{8,9,11}$ using only the term proportional to $\partial T_{c} / \partial n$.

\section{CONCLUSIONS}

In conclusion, we have shown that the Bernoulli potential in superconductors can be discussed by the charge build up in the region between the surface and $\max [\xi / \sqrt{2}, \lambda / 2]$. The thinner depleted charge region carries the surface charge. In contrast to former theories which assumed that the surface charge is localized on the scale of Thomas-Fermi screening length $\lambda_{\mathrm{TF}}$, we find that the surface charge extends over a range $L=\min [\xi / \sqrt{2}, \lambda / 2]$. In fact, there is a nonzero contribution on the scale of $\lambda_{\mathrm{TF}}$, but of a negligibly small amplitude proportional to $\lambda_{\mathrm{TF}}^{2} / L^{2}$.

For extreme type-II superconductors we have confirmed the picture known already from London with the thermodynamic corrections by Rickayzen. The bulk charge extends on the scale of the London penetration depth $\lambda / 2$. The surface charge is localized on the scale of the GL coherence length $\xi / \sqrt{2}$ which is negligible in this limit anyway. This is in agreement with the classical treatment which neglects all gradient contributions.

In type-I or weak type-II superconductors, i.e for the GL parameter $\kappa<\sqrt{2}$, one finds the opposite situation. The surface charge is localized on the scale of the London penetration depth $\lambda$ while the bulk extends on the scale of the GL coherence length $\xi / \sqrt{2}$. In this case the gradient contributions are dominant and local London-type theories naturally fail.

In strong magnetic fields the Bernoulli potential becomes a non-quadratic function of the magnetic field. This feature parallels the nonlinear susceptibility as it is dominated by the enhanced penetration of strong magnetic fields into superconductors. There is an additional thermodynamic correction absent in low magnetic fields, however, for realistic material parameters (like for niobium) it accounts for less than $3 \%$ of the charge density. Thus the approximations used in ${ }^{8,9,11}$ are justified.

Finally we remind that the potential $\phi$ discussed here does not include the surface dipole which affects the potential seen outside the sample. We will discuss this contribution in a forthcoming paper.

\section{ACKNOWLEDGMENTS}

This work was supported by MŠMT program Kontakt ME601 and GAČR 202/03/0410, GAAV A1010312 grants. The European (ESF) program VORTEX is also acknowledged.
${ }^{1}$ F. Bopp, Z. Phys. 107, 623 (1937).

${ }^{2}$ F. London, Superfluids (Wiley, New York, 1950), Vol. I, Sec. 8.

${ }^{3}$ A. G. van Vijfeijken and F. S. Staas, Phys. Lett. 12, 175 (1964).

${ }^{4}$ E. Jakeman and E. R. Pike, Proc. Phys. Soc. 91, 422 (1967).

${ }^{5}$ C. J. Adkins and J. R. Waldram, Phys. Rev. Lett. 21, 76 (1968).

${ }^{6}$ G. Rickayzen, J. Phys. C 2, 1334 (1969).

${ }^{7}$ K. E. Gray (ed.), Nonequilibrium Superconductivity, Phonons, and Kapitza Boundaries (Plenum NY, 1981)

${ }^{8}$ D. I. Khomskii and F. V. Kusmartsev, Phys. Rev. B 46, 14245 (1992).

${ }^{9}$ D. I. Khomskii and A. Freimuth, Phys. Rev. Lett. 75, 1384 (1995).

${ }^{10}$ D. van der Marel, Physica C 165, 35 (1990).

${ }^{11}$ G. Blatter et al, Phys. Rev. Lett. 77, 566 (1996).

12 M. A. R. LeBlanc, Supercond. Sci. Technol. 10, 929 (1997).

13 J. Koláček, P. Lipavský and E. H. Brandt, Phys. Rev. Lett. 86, 312 (2001).

${ }^{14}$ J. Koláček and P. Lipavský, Physica C 364-365, 138 (2001).

${ }^{15}$ P. Lipavský, J. Koláček, K. Morawetz and E. H. Brandt, Phys. Rev. B 65, 144511 (2002).

${ }^{16}$ S. V. Yampolskii, B. J. Baelus, F. M. Peeters and J. Koláček, Phys. Rev. B 64, 144511 (2001).

17 J. Koláček, P. Lipavský and E. H. Brandt, Physica C 369 , 55 (2002).

18 J. Bardeen, Theory of Superconductivity in Handbuch der Physik, Bd. XV, 274 (Springer Berlin, 1956).

${ }^{19}$ C. C. Koch, J. O. Scarbrough and D. M. Kroeger, Phys. Rev. B 9, 888 (1974). 